\title{
Towards the three loop evolution equation for GPDs
}

\author{
A. N. Manashov* \\ Institut für Theoretische Physik, Universität Hamburg, D-22761 Hamburg, Germany \\ E-mail: alexander.manashov@desy.de
}

QCD in $d=4-2 \varepsilon$ dimensions and large number of flavors possesses a nontrivial infrared stable critical point. At the critical point the theory is invariant under the conformal transformations. It implies that the evolution kernel of leading-twist light-ray operators commutes with the generators of conformal transformations. Taking into account that the evolution kernels in the MS-scheme are not sensitive to the space-time dimensions one concludes that QCD evolution equations in MS-schemes have a hidden symmetry. Namely, the evolution kernel commutes with the generators of the conformal algebra. The explicit form of the generators differs from their canonical (classical) form and can be derived by studying conformal Ward identities. Invariance with respect to the conformal transformations imposes nontrivial constraints on the form of evolution kernels and allows one to restore the nonforward evolution kernels for the nonsinglet operators from the known NNLO anomalous dimensions. We present here the two loop expressions for the generators of conformal algebra.

QCD Evolution 2016

May 30-June 03, 2016

National Institute for Subatomic Physics (Nikhef), Amsterdam

${ }^{*}$ Speaker. 


\section{Introduction}

The recent progress in the experimental particle physics due to advances in accelerator and detector technologies has made possible the study of hard exclusive reactions - reactions with identified particles in the final state. The theoretical description of such processes is based on the QCD factorization theorems and Operator Product Expansion (OPE). The transition amplitude for a particular scattering processes can be represented as a convolution of nonperturbative functions so-called generalized parton distributions (GPDs), or distribution amplitudes (DAs) with a coefficient functions that can be calculated in perturbation theory. Scale dependence of the GPDs (DAs) is governed by the renormalization group equations (RGEs). For processes with nonzero momentum transfer from initial to the final state one has to keep under control the mixing of operators with a different number of total derivatives. The matrix of anomalous dimensions has a triangular form with the diagonal entries equal to the anomalous dimensions that are known nowadays to NNLO accuracy $[1,2]$. The nondiagonal entries, however, require a dedicated calculation.

Despite continuous progress in the multiloop calculation technique the direct calculation of the mixing coefficients in higher orders is quite challenging. However, it has been known for some time [3] that conformal symmetry of QCD Lagrangian imposes certain restrictions on the form of the mixing matrix. This allows one to resolve mixing at given order of perturbation theory performing an additional calculation at one order less. This technique was used to compute the nonforward evolution kernels for the twist-two operators in QCD [4, 5, 6, 7, 8, 9, 10]. with two-loop accuracy.

A different approach to this problem was suggested in [11]. The idea is to take advantage of the existence of the nontrivial Wilson-Fisher fixed point of QCD in $d=4-2 \varepsilon$ dimensions. It is well known that at a (critical) point the Poincare symmetry of a theory is enhanced, as a rule, to include the scale and conformal transformations (see Ref. [12] for a review). It is expected that in a conformal theory the RG kernels will commute with the generators of the conformal group. However, due to quantum corrections the form of the generators differ from their canonical expression. Finally, noticing that in the MS-like scheme the RG kernels (anomalous dimensions matrices) are not sensitive to the space-time dimension one deduces that the RG kernels are essentially the same in the physical four dimensional theory and $d=4-2 \varepsilon$ theory at the critical point.

The utility of this approach was demonstrated on several examples in Refs. [11,13]. Below we discuss the details of the two-loop calculation of the generator of special conformal transformations presented Ref. [14].

\section{QCD in $d=4-2 \varepsilon$ dimensions}

It is well known that $\mathrm{QCD}$ in $d=4-2 \varepsilon$ dimension possesses a nontrivial critical point for a large number of quark flavors ${ }^{1}$. Indeed, the $\beta$-function in the $d$-dimensional theory takes the form

$$
\beta(a)=M \frac{d a}{d M}=2 a\left(-\varepsilon-\gamma_{g}\right)
$$

\footnotetext{
${ }^{1}$ This property was used to develop the $1 / N_{f}$ expansion for the RG functions in QCD $[15,16,17]$.
} 
where $a=\alpha_{s} / 4 \pi$ and

$$
\gamma_{g}=\beta_{0} a+\beta_{1} a^{2}+\mathscr{O}\left(a^{3}\right)
$$

Taking into account that

$$
\beta_{0}=\frac{11}{3} N_{c}-\frac{2}{3} N_{f}, \quad \quad \beta_{1}=\frac{2}{3}\left[17 N_{c}^{2}-5 N_{c} N_{f}-3 C_{F} N_{f}\right]
$$

one concludes that the $\beta$ function vanishes $\left(\beta\left(a_{*}\right)=0\right)$ for

$$
a_{*}(\varepsilon)=-\frac{\varepsilon}{\beta_{0}}-\left(\frac{\varepsilon}{\beta_{0}}\right)^{2} \frac{\beta_{1}}{\beta_{0}}+O\left(\varepsilon^{3}\right) .
$$

The vanishing of the $\beta$ functions implies the conformal symmetry of the theory [12]. In gauge theories the situation is a more subtle - one can expect only that the correlation functions of local gauge-invariant operators transform in a proper way under conformal transformations. Notice also that for the quantities of interest the dependence on $N_{f}$ is always polynomial so that the requirement for $N_{f}$ to be large can be relaxed.

Let $\left\{\mathscr{O}_{i}, i=1, \ldots, n\right\}$ be a set of the local leading twist operators of a given (canonical) dimension. These operators mix under renormalization and the RG equation takes the matrix form

$$
\left(\left(M \partial_{M}+\beta(a) \partial_{a}\right) \delta_{i k}+\gamma_{i k}(a)\right) \mathscr{O}_{i}=0,
$$

where $\gamma_{i k}$ is the anomalous dimension matrix. The form of the matrix $\gamma_{i k}$ depends strongly on the choice of the basis operators, $\mathscr{O}_{i}$. The symmetry properties of the problem are not transparent in this formulation. The symmetry transformations take the simplest form in the coordinate space. Therefore it is preferable to switch to a different description of the operators where the symmetry generators are realized in the simplest way. Such a description is given by the so-called light-ray operators [18] which are the generating functions for the local ones. The twist-two nonsinglet light-ray operator takes the form

$$
[\mathscr{O}]\left(x ; z_{1}, z_{2}\right)=Z \mathscr{O}\left(x ; z_{1}, z_{2}\right)=Z \bar{q}\left(x+z_{1} n\right) \not h q\left(x+z_{2} n\right)=\sum_{m, k} \frac{z_{1}^{m} z_{2}^{k}}{m ! k !}\left[\bar{q}(x) \overleftarrow{D_{+}{ }^{m}} h \vec{h}_{+}^{k} q(x)\right]
$$

where $D_{+}=n^{\mu} D_{\mu}$ and the gauge link between the quark fields is tacitly assumed. The square brackets stand for the renormalization in the $\overline{\mathrm{MS}}$ scheme. The renormalization factor $Z$ is now an integral operator acting on the light-cone coordinates $z_{1}, z_{2}$

$$
Z=1+\sum_{k=0}^{\infty} \varepsilon^{-k} Z_{k}(a), \quad Z_{k}(a)=\sum_{\ell=k}^{\infty} a^{\ell} Z_{k}^{(\ell)} .
$$

The RGE for the light-ray operator $[\mathscr{O}]$ takes the form

$$
\left(M \partial_{M}+\beta(a) \partial_{a}+\mathbb{H}(a)\right)[\mathscr{O}]\left(x ; z_{1}, z_{2}\right)=0 .
$$

The integral operator $\mathbb{H}$ is related to the renormalization factor (2.7) as follows

$$
\mathbb{H}(a)=2 \gamma_{q}(a)-M \frac{d}{d M} Z Z^{-1}=2 \gamma_{q}(a)+2 \sum_{\ell=1}^{\infty} \ell a^{\ell} Z_{1}^{(\ell)}=\sum_{k \geq 1} a^{k} \mathbb{H}^{(k)}
$$


$\gamma_{q}$ is the quark anomalous dimension. It can be shown that the evolution kernel can be written as follows

$$
\mathbb{H}(a)[\mathscr{O}]\left(z_{1}, z_{2}\right)=\int_{0}^{1} d \alpha \int_{0}^{1} d \beta h(\alpha, \beta)[\mathscr{O}]\left(z_{12}^{\alpha}, z_{21}^{\beta}\right),
$$

where $z_{12}^{\alpha}=z_{1} \bar{\alpha}+z_{2} \alpha$ and $\bar{\alpha}=1-\alpha$. The perturbative series for the weight function $h(\alpha, \beta)$ has the form

$$
h(\alpha, \beta)=a h^{(1)}(\alpha, \beta)+a^{2} h^{(2)}(\alpha, \beta)+\ldots,
$$

where the fixed-order kernels $h^{(k)}(\alpha, \beta)$ in the $\overline{\mathrm{MS}}$ scheme do not depend on $\varepsilon$ by construction. Thus all dependence of the kernel $\mathbb{H}$ at the critical point on the space-time dimension $d$ comes only via the critical coupling, $a_{*}=a_{*}(\varepsilon)$

$$
\mathbb{H}\left(a_{*}\right)=a_{*} \mathbb{H}^{(1)}+a_{*}^{2} \mathbb{H}^{(2)}+\ldots
$$

It follows from these considerations that the RGEs in four dimensional theory in the $\overline{\mathrm{MS}}$-like scheme inherit all symmetries of the evolution equations in the conformal theory in non-integer dimensions.

\section{Symmetry generators}

At the critical point the theory enjoys the conformal symmetry and the evolution kernel $\mathbb{H}\left(a_{*}\right)$ commutes with the generators of the conformal transformations. Only three of all generators act non-trivially on the twist-two light ray operator. These transformation belongs to the so-called soft-collinear $S L(2, R)$ subgroup of the conformal group. The canonical (the tree level) generators, $S_{\alpha}=\left\{S_{+}, S_{0}, S_{-}\right\}$, are given by well known expressions

$$
S_{+}^{(0)}=z_{1}^{2} \partial_{z_{1}}+z_{2}^{2} \partial_{z_{2}}+2\left(z_{1}+z_{2}\right), \quad S_{0}^{(0)}=z_{1} \partial_{z_{1}}+z_{2} \partial_{z_{2}}+2, \quad S_{-}^{(0)}=-\partial_{z_{1}}-\partial_{z_{2}} .
$$

Beyond the leading order two of the generators, $S_{0}$ and $S_{+}$are modified by quantum corrections,

$$
S_{0}=S_{0}^{(0)}+\Delta S_{0}, \quad S_{+}=S_{+}^{(0)}+\Delta S_{+},
$$

but still satisfy the $S L(2)$ commutation relations

$$
\left[S_{+}, S_{-}\right]=2 S_{0}, \quad\left[S_{0}, S_{ \pm}\right]= \pm S_{ \pm} .
$$

The form of the operator $S_{0}$ can be fixed from general considerations,

$$
S_{0}=S_{0}^{(0)}+\gamma_{g}\left(a_{*}\right)+\frac{1}{2} \mathbb{H}\left(a_{*}\right) .
$$

The first of the commutation relations (3.3) allows one to determine the generator of special conformal transformations, $S_{+}$, up to one unknown functions $\Delta_{+}$,

$$
S_{+}=S_{+}^{(0)}+\left(z_{1}+z_{2}\right)\left(\gamma_{g}\left(a_{*}\right)+\frac{1}{2} \mathbb{H}\left(a_{*}\right)\right)+\left(z_{1}-z_{2}\right) \Delta_{+}\left(a_{*}\right)
$$


The operator $\Delta_{+}$(conformal anomaly) commutes with translations, $\left[S_{-}, \Delta_{+}\right]=0$, and with the canonical generator $S_{0}^{(0)}$. These properties imply that the operator $\Delta_{+}$can be represented in the form (2.10).

The second of the commutation relations (3.3), $\left[S_{0}, S_{+}\right]=S_{+}$, gives rise to the following equation for the evolution kernel, $\left[S_{+}, \mathbb{H}\left(a_{*}\right)\right]=0$, that results in a nontrivial relation between $\mathbb{H}$ and $\Delta_{+}$. Namely,

$$
\left[S_{+}^{(0)}, \mathbb{H}\left(a_{*}\right)\right]=\left[\mathbb{H}\left(a_{*}\right), z_{1}+z_{2}\right]\left(\gamma_{g}\left(a_{*}\right)+\frac{1}{2} \mathbb{H}\left(a_{*}\right)\right)+\left[\mathbb{H}\left(a_{*}\right),\left(z_{1}-z_{2}\right) \Delta_{+}\left(a_{*}\right)\right] .
$$

Expanding Eq. (3.6) in a series in $a_{*}$ one gets

$$
\begin{aligned}
{\left[S_{+}^{(0)}, \mathbb{H}^{(1)}\right]=} & 0 \\
{\left[S_{+}^{(0)}, \mathbb{H}^{(2)}\right]=} & {\left[\mathbb{H}^{(1)}, z_{1}+z_{2}\right]\left(\gamma_{g}^{(1)}+\frac{1}{2} \mathbb{H}^{(1)}\right)+\left[\mathbb{H}^{(1)},\left(z_{1}-z_{2}\right) \Delta_{+}^{(1)}\right] } \\
{\left[S_{+}^{(0)}, \mathbb{H}^{(3)}\right]=} & {\left[\gamma_{g}^{(2)} \mathbb{H}^{(1)}+\gamma_{g}^{(1)} \mathbb{H}^{(2)}, z_{1}+z_{2}\right]+\frac{1}{2}\left[\mathbb{H}^{(2)}, z_{1}+z_{2}\right] \mathbb{H}^{(1)}+\frac{1}{2}\left[\mathbb{H}^{(1)}, z_{1}+z_{2}\right] \mathbb{H}^{(2)} } \\
& +\left[\mathbb{H}^{(2)},\left(z_{1}-z_{2}\right) \Delta_{+}^{(1)}\right]+\left[\mathbb{H}^{(1)},\left(z_{1}-z_{2}\right) \Delta_{+}^{(2)}\right]
\end{aligned}
$$

and so on. Since the kernel $\mathbb{H}\left(a_{*}\right)$ commutes with $S_{-}^{(0)}$ and $S_{0}^{(0)}$ (it commutes with the full generators $S_{-}, S_{0}$ as well) these equations demonstrate that the non-invariant part of the $\ell$-loop kernel (with respect to the canonical conformal transformations) is completely fixed by the lower order kernels $\mathbb{H}^{(k)}, \Delta_{+}^{(k)}, k=1, \ldots, \ell-1$. The explicit form of the operator $\Delta_{+}$can be derived from analysis of the conformal Ward Identity (CWI) [8, 10, 14].

\section{Conformal generators at two loops}

In order to fix the form of the generator $S_{+}$it is convenient to consider the correlator of the two light-ray operators,

$$
\mathscr{G}(x ; z, w)=\left\langle\left[\mathscr{O}^{(n)}\right](0, z)\left[\mathscr{O}^{(\bar{n})}\right](x, w)\right\rangle=\mathscr{N} \int D \Phi e^{-S_{R}(\Phi)}\left[\mathscr{O}^{(n)}\right](0, z)\left[\mathscr{O}^{(\bar{n})}\right](x, w) .
$$

It can be shown that this correlator satisfies the following equation [14]

$$
\begin{aligned}
\frac{i}{2} \bar{n}_{\mu}\left(\left\langle\delta_{K}^{\mu}\left[\mathscr{O}^{(n)}\right](0, z)\left[\mathscr{O}^{(\bar{n})}\right](x, w)\right\rangle+\left\langle\left[\mathscr{O}^{(n)}\right](0, z)\right.\right. & \left.\left.\left.\delta_{K}^{\mu}\left[\mathscr{O}^{(\bar{n})}\right](x, w)\right]\right\rangle\right)= \\
& =\left[(n \bar{n}) S_{+}^{(z)}-\frac{1}{2} x^{2}\left(\bar{n} \partial_{x}\right)\right] \mathscr{G}(x ; z, w)=0,
\end{aligned}
$$

where $z=\left\{z_{1}, z_{2}\right\}, w=\left\{w_{1}, w_{2}\right\}$ and we assume that $(x \cdot n)=(x \cdot \bar{n})=0$. This equation can be considered as the defining relation for the generator $S_{+}$. In order to find the explicit form of $S_{+}$one can consider CWI for the correlator (4.1). It follows from invariance of the integral (4.1) under the change of variables:

$$
\Phi \mapsto \Phi+\delta_{K}^{\mu} \Phi, \quad \delta_{K}^{\mu} \Phi=\left(2 x_{\mu}(x \partial)-x^{2} \partial_{\mu}+2 \Delta_{\Phi} x_{\mu}-2 x^{v} \Sigma_{\mu v}\right) \Phi(x) .
$$


Here $\Sigma_{\mu \nu}$ is the generator of spin rotations,

$$
\Sigma_{\mu \nu} c=\Sigma_{\mu \nu} \bar{c}=0, \quad \Sigma_{\mu \nu} q=\frac{i}{2} \sigma_{\mu \nu} q, \quad \Sigma_{\mu v} A_{\alpha}=g_{v \alpha} A_{\mu}-g_{\mu \alpha} A_{v},
$$

$\Delta_{\Phi}$ is the dimension of the corresponding field. It is convenient to choose [10]:

$$
\Delta_{q}=\frac{3}{2}-\varepsilon, \quad \Delta_{A}=1, \quad \Delta_{c}=0, \quad \Delta_{\bar{c}}=2-\varepsilon .
$$

The CWI takes the form

$$
\left\langle\delta\left[\mathscr{O}^{(n)}\right](0, z)\left[\mathscr{O}^{(\bar{n})}\right](x, w)\right\rangle+\left\langle\left[\mathscr{O}^{(n)}\right](0, z) \delta\left[\mathscr{O}^{(\bar{n})}\right](x, w)\right\rangle=\left\langle\delta S_{R}\left[\mathscr{O}^{(n)}\right](0, z)\left[\mathscr{O}^{(\bar{n})}\right](x, w)\right\rangle,
$$

where $\delta=\bar{n}_{\mu} \delta_{K}^{\mu}$, and $\delta S_{R}$ is the variation of the QCD action (in Euclidean space)

$$
\delta_{K}^{\mu} S_{R}=\int d^{d} x 2 x^{\mu}\left(\mathscr{N}(x)-(d-2) \partial^{\rho} \mathscr{B}_{\rho}(x)\right) .
$$

The operators $\mathscr{N}$ and $\mathscr{B} \rho$ are given by the following expressions

$$
\mathscr{N}(x)=2 \varepsilon \mathscr{L}_{R}^{Y M+g f}=2 \varepsilon\left(\frac{1}{4} Z_{A}^{2} F^{2}+\frac{1}{2 \xi}(\partial A)^{2}\right), \quad \mathscr{B}_{\rho}(x)=Z_{c}^{2} \bar{c} D^{\rho} c-\frac{1}{\xi} A^{\rho}(\partial A) .
$$

Let us note that in distinction with the non-gauge theories the variation (4.6) does not vanish even at $d=4$. However, the non-vanishing term, $\partial^{\rho} \mathscr{B}_{\rho}$, being the BRST variation does not contribute to the correlation functions of gauge invariant operators ${ }^{2}$. The further analysis goes along the lines of Ref. [20]. One reexpands the operator $\mathscr{N}$ over the set of the renormalized (finite) operators,

$$
\mathscr{N}=-\frac{\beta(a)}{a}\left[\mathscr{L}^{Y M+g f}\right]-\left(\gamma_{A}+\gamma_{g}\right) \Omega_{A}-\sum_{\Phi \neq A} \gamma_{\Phi} \Omega_{\Phi}+\frac{\gamma_{A}}{\xi}\left[(\partial A)^{2}\right]+z_{c} \partial^{\mu} \Omega_{\mu}+z_{b} \partial_{\mu}\left[\mathscr{B}^{\mu}\right] .
$$

The r.h.s. of this equation contains EOM operators, $\Omega_{\Phi}=\Phi(y)\left(\delta S_{R} / \delta \Phi(y)\right), \partial^{\mu} \Omega_{\mu}=\Omega_{\bar{c}}-\Omega_{c}$, the BRST variation, $\left[\mathscr{B}^{\mu}\right]$, and two renormalized operators $\left[\mathscr{L}^{Y M+g f}\right]$ and $\left[(\partial A)^{2}\right]$. All of them enter with the finite coefficients $-\gamma_{\Phi}-$ the field anomalous dimensions, $z_{c}(g, \xi)$ and $z_{b}(g, \xi)$. The operator $\left[\mathscr{B}^{\mu}\right]$ and the ghost EOM do not contribute to the correlator (4.5). It can be shown that that quark and gluon EOM terms together with the gauge fixing term give rise to the expected modification of the scaling dimensions in the expression for the conformal generator. Thus all nontrivial corrections to the generator $S_{+}$originate from the first term on the r.h.s of (4.8). Although this term appears with the coefficient $\beta(a) / a$ which vanishes at the critical point the correlator

$$
\left\langle\int d^{d} y \mathscr{N}(y)\left[\mathscr{O}^{(n)}\right](0, z)\left[\mathscr{O}^{(\bar{n})}\right](x, w)\right\rangle
$$

contains additional UV divergencies. They arise from the integration regions where the arguments of the renormalized operators close to each other: $y \sim 0$ or $y \sim x$. Thus the correlator (4.9) requires additional renormalization that gives rise to a nonzero contribution to CWI from this term.

It can be shown that for the given choice of the vectors $x, n, \bar{n}$ all corrections to $S_{+}$come only from pair counterterms to the operators $\mathscr{N}(y)\left[\mathscr{O}^{(n)}\right](0, z) \sim \delta(y) Z(\varepsilon)\left[\mathscr{O}^{(n)}\right](0, z)$. Technical details are rather lengthy and can be found in Ref. [14]. Here we give only the final answer.

\footnotetext{
${ }^{2}$ It explains why the correlator $\langle\mathscr{O} q \bar{q}\rangle$, is not convenient object for analysis in the case of gauge theories.
} 
One loop correction to the generator $S_{+}$takes a very simple form $[8,13]$

$$
\Delta_{+}^{(1)} f\left(z_{1}, z_{2}\right)=2 C_{F} \int_{0}^{1} d \alpha \int_{0}^{1} d u \frac{\bar{\alpha}}{\alpha}\left[f\left(z_{12}^{\alpha u}, z_{2}\right)-f\left(z_{1}, z_{21}^{\alpha u}\right)\right] .
$$

At two loop the correction can be written [14] as follows

$$
z_{12} \Delta_{+}^{(2)}=z_{12} \Delta^{(2)}+\frac{1}{4}\left[\mathbb{H}^{(2)}, z_{1}+z_{2}\right]
$$

where the operator $\Delta^{(2)}$ has the form

$$
\begin{aligned}
\Delta^{(2)} f\left(z_{1}, z_{2}\right)= & \int_{0}^{1} d \alpha \int_{0}^{\bar{\alpha}} d \beta\left[\omega(\alpha, \beta)+\omega^{\mathbb{P}}(\alpha, \beta) \mathbb{P}_{12}\right]\left[f\left(z_{12}^{\alpha}, z_{21}^{\beta}\right)-f\left(z_{12}^{\beta}, z_{21}^{\alpha}\right)\right] \\
& +\int_{0}^{1} d u \int_{0}^{1} d t \varkappa(t)\left[f\left(z_{12}^{u t}, z_{2}\right)-f\left(z_{1}, z_{21}^{u t}\right)\right] .
\end{aligned}
$$

Here $\mathbb{P}_{12} f\left(z_{1}, z_{2}\right)=f\left(z_{2}, z_{1}\right)$ is the permutation operator and the kernels $\varkappa(t), \omega(\alpha, \beta), \omega^{\mathbb{P}}(\alpha, \beta)$ receive contributions of three different color structures

$$
\begin{aligned}
\varkappa(t) & =C_{F}^{2} \varkappa_{\mathrm{P}}(t)+\frac{C_{F}}{N_{C}} \varkappa_{F A}(t)+C_{F} \beta_{0} \varkappa_{b F}(t), \\
\omega(\alpha, \beta) & =C_{F}^{2} \omega_{\mathrm{P}}(\alpha, \beta)+\frac{C_{F}}{N_{C}} \omega_{F A}(\alpha, \beta), \\
\omega^{\mathbb{P}}(\alpha, \beta) & =\frac{C_{F}}{N_{C}} \omega_{F A}^{\mathbb{P}}(\alpha, \beta),
\end{aligned}
$$

The explicit expressions for the kernels $\chi, \omega$ are

$$
\begin{aligned}
\varkappa_{b F}(t)= & -2 \frac{\bar{t}}{t}\left(\ln \bar{t}+\frac{5}{3}\right) \\
\varkappa_{F A}(t)= & \frac{2 \bar{t}}{t}\left\{(2+t)\left[\operatorname{Li}_{2}(\bar{t})-\mathrm{Li}_{2}(t)\right]-(2-t)\left(\frac{t}{\bar{t}} \ln t+\ln \bar{t}\right)-\frac{\pi^{2}}{6} t-\frac{4}{3}-\frac{t}{2}\left(1-\frac{t}{\bar{t}}\right)\right\} \\
\varkappa_{\mathrm{P}}(t)= & 4 \bar{t}\left[\operatorname{Li}_{2}(\bar{t})-\mathrm{Li}_{2}(1)\right]+4\left(\frac{t^{2}}{\bar{t}}-\frac{2 \bar{t}}{t}\right)\left[\operatorname{Li}_{2}(t)-\mathrm{Li}_{2}(1)\right]-2 t \ln t \ln \bar{t}-\frac{\bar{t}}{t}(2-t) \ln ^{2} \bar{t} \\
+ & \frac{t^{2}}{\bar{t}} \ln ^{2} t-2\left(1+\frac{1}{t}\right) \ln \bar{t}-2\left(1+\frac{1}{\bar{t}}\right) \ln t-\frac{16}{3} \bar{t}-1-5 t \\
\omega_{\mathrm{P}}(\alpha, \beta)= & \frac{4}{\alpha}\left[\operatorname{Li}_{2}(\bar{\alpha})-\operatorname{Li}_{2}(1)\right]+\frac{1}{\alpha} \ln ^{2} \bar{\alpha}-(\alpha-\beta) \ln ^{2}\left(\frac{\alpha}{\bar{\alpha}}\right)-\beta \ln { }^{2} \alpha \\
& +2 \alpha\left(\frac{\pi^{2}}{3}-\frac{15}{2}\right)-2\left(\alpha+\beta+\frac{1}{\bar{\alpha}}\right) \ln \alpha+(\beta-2 \bar{\alpha})\left(1+\frac{2}{\alpha}\right) \ln \bar{\alpha} \\
\omega_{F A}(\alpha, \beta)= & 2\left[\left(\frac{1}{\alpha}-\alpha\right)\left[\operatorname{Li}_{2}\left(\frac{\beta}{\bar{\alpha}}\right)-\operatorname{Li}_{2}(\beta)-2 \operatorname{Li} 2(\alpha)-\ln \alpha \ln \bar{\alpha}\right]+\frac{\alpha}{\tau}(\tau \ln \tau+\bar{\tau} \ln \bar{\tau})\right. \\
& \left.-\bar{\beta} \ln \alpha-\frac{\bar{\alpha}}{\alpha} \ln \bar{\alpha}\right], \\
\omega_{F A}^{\mathbb{P}}(\alpha, \beta)= & 2\left[\left(\bar{\alpha}-\frac{1}{\bar{\alpha}}\right)\left[\operatorname{Li}_{2}\left(\frac{\alpha}{\bar{\beta}}\right)-\operatorname{Li}_{2}(\alpha)-\ln \bar{\alpha} \ln \bar{\beta}\right]+\alpha \bar{\tau} \ln \bar{\tau}+\frac{\beta^{2}}{\bar{\beta}} \ln \bar{\alpha}\right]
\end{aligned}
$$

where $\tau=\alpha \beta / \bar{\alpha} \bar{\beta}$. 


\section{Acknowledgments}

The author thanks V.M. Braun, S. Moch and M. Strohmaier for the collaboration on this project. This work was supported by Deutsche Forschungsgemeinschaft (DFG) with the grant MO 1801/1-1.

\section{References}

[1] S. Moch, J. A. M. Vermaseren and A. Vogt, The Three loop splitting functions in QCD: The Nonsinglet case, Nucl. Phys. B 688 (2004) 101.

[2] A. Vogt, S. Moch and J. A. M. Vermaseren, The Three-loop splitting functions in QCD: The Singlet case, Nucl. Phys. B 691 (2004) 129.

[3] D. Müller, Constraints for anomalous dimensions of local light cone operators in $\phi^{3}$ in six-dimensions theory, Z. Phys. C 49 (1991) 293.

[4] D. Müller, Conformal constraints and the evolution of the nonsinglet meson distribution amplitude, Phys. Rev. D 49 (1994) 2525.

[5] D. Müller, Restricted conformal invariance in QCD and its predictive power for virtual two photon processes, Phys. Rev. D 58 (1998) 054005.

[6] D. Mueller, Scheme dependence of NLO corrections to exclusive processes, Phys. Rev. D 59 (1999) 116003.

[7] A. V. Belitsky and D. Müller, Predictions from conformal algebra for the deeply virtual Compton scattering, Phys. Lett. B 417 (1998) 129.

[8] A. V. Belitsky and D. Müller, Next-to-leading order evolution of twist-2 conformal operators: The Abelian case, Nucl. Phys. B 527 (1998) 207.

[9] A. V. Belitsky, A. Freund and D. Müller, Evolution kernels of skewed parton distributions: Method and two loop results, Nucl. Phys. B 574 (2000) 347.

[10] A. V. Belitsky and D. Müller, Broken conformal invariance and spectrum of anomalous dimensions in QCD, Nucl. Phys. B 537 (1999) 397.

[11] V. M. Braun and A. N. Manashov, Evolution equations beyond one loop from conformal symmetry, Eur. Phys. J. C 73 (2013) 2544.

[12] Y. Nakayama, Scale invariance vs conformal invariance, Phys. Rept. 569 (2015) 1.

[13] V. M. Braun and A. N. Manashov, Two-loop evolution equations for light-ray operators, Phys. Lett. B 734 (2014) 137.

[14] V. M. Braun, A. N. Manashov, S. Moch and M. Strohmaier, Two-loop conformal generators for leading-twist operators in QCD, JHEP 1603 (2016) 142

[15] J. A. Gracey, The QCD Beta function at $O\left(1 / N_{f}\right)$, Phys. Lett. B 373 (1996) 178.

[16] J. A. Gracey, Anomalous dimension of nonsinglet Wilson operators at $O\left(1 / N_{f}\right)$ in deep inelastic scattering, Phys. Lett. B 322 (1994) 141.

[17] M. Ciuchini, S. E. Derkachov, J. A. Gracey and A. N. Manashov, Quark mass anomalous dimension at $O\left(1 / N_{f}^{2}\right)$ in $Q C D$, Phys. Lett. B 458 (1999) 117. 
[18] I. I. Balitsky and V. M. Braun, Evolution Equations for QCD String Operators, Nucl. Phys. B 311 (1989) 541.

[19] V. M. Braun, G. P. Korchemsky and D. Müller, The Uses of conformal symmetry in QCD, Prog. Part. Nucl. Phys. 51 (2003) 311.

[20] A. N. Vasil'ev, The field theoretic renormalization group in critical behavior theory and stochastic dynamics, Boca Raton, USA: Chapman \& Hall/CRC (2004) 681 p. 